\title{
Understanding Consumers' Reactance of Online Personalized Advertising: from a Perspective of Negative Effects
}

\author{
Qi Chen \\ Harbin Institute of \\ Technology \\ chenqi1208@126.com
}

\author{
Yuqiang Feng \\ Harbin Institute of \\ Technology \\ fengyq@ hit.edu.cn
}

\author{
Luning Liu \\ Harbin Institute of \\ Technology \\ liuluning830730@gmail.com
}

\author{
Jingrui Ju \\ Harbin Institute of \\ Technology \\ jirrui@sina.cn
}

\begin{abstract}
Despite the increasing popularity of IT-enabled personalization, the online consumers' attitude of reactance appears to be a major inhibiting result in their acceptance of the online personalized advertising. The objective of this study is to study consumers' reactance of online personalized advertising from the perspective of negative effects. Especially, we identify the rational choice factors rooted in the rational choice theory in the context of reactance and test their impacts on reactance, with consideration of individual feeling factors in a specific situation of personalization paradox. We also identify the contingent effects of consumers' goals. By analyzing the survey data from 281 respondents, our results indicate that the curiosity and vulnerability significantly impact on the rational choice factors, and the influences of the rational choice factors on consumers' reactance vary in the context of consumers' different goals (searching and browsing). Theoretical and practical implications are also discussed.
\end{abstract}

\section{Introduction}

With the rapid development of cloud computing, mobile payment and social media, IT-enabled personalization has been defined as one of the best ways for the companies to improve their profitability and better consumers' responsiveness by exploiting consumer data to influence purchase decisions [1]. Many online firms such as Tmall and Amazon collect users' data, and then use the collection to implement online personalized advertising on their platforms. Consumers might perceive these services as more attractive and favorite [2].

However, the personalized advertising services can also cause consumers' unfavorable responses [3]. As an example, the 2013 Choicestream Survey with 1,042 completed surveys announced that "only $13 \%$ of consumers admitted to clicking on one of these retargeting ads". Compared with the number of the personalized online advertisements saw by each consumer per day, the results of click-through rates point to a low success for any one campaign [4]. To consumers, on the one hand, without such services, the consumers might be trapped with cognitive overload and complex consumption [5], while on the other hand, when receiving personalized advertising services, they might be turned away by privacy concern because of their personal information instinctly analyzed, used or shared in consumer transactions with the online retailers. Moreover, the online personalized advertising only presents to the consumers the things they seem like, which might inhibit their capacity to decide what they choose, what they buy and even what they think [6]. This issue has given rise to a personalization paradox, where the benefits from the personalized online advertising services may come at sacrifices of taking on greater risks of privacy concern and the only focus on this service with little attention to other alternative service offerings.

Therefore, it is not surprising that a large amount of consumers choose to ignore the personalized advertisements with psychological reactance, when such personalized services follow them after they leave the IT platforms [7]. Reactance is a psychological state motivated by consumers to resist something when they find coercive or threatened of their freedom by behaving in the opposite way to that intended [8]. When the consumer experience reactance in such personalization advertising services, they may "avoid complying with a persuasion attempt but does not directly characterize the cause of discomfort associated with a privacy invasion" ([7], P2). In particular, consumers' different reasons for surfing the IT platforms might contribute to various beliefs towards IT-enabled services [9]. For example, the consumers who intent to browse for entertainment would hold less trust beliefs towards the ITenabled services than those whose goals are to search useful information [9]. Correspondingly, the outcomes of the personalized online advertising services are sometimes negative varying upon the consumers' various goals to surf the IT platforms (e.g. searching and browsing).

It is assumed that consumers often act as rational economic agents in regarding to the personalization paradox, who consider the benefits of personalization and the costs of related risks [10]. Hence, our study intends to extend the knowledge about the personalization paradox from the perspective of negative effects by combining the rational choice factors and feeling factors. In doing so, our study addresses three questions:

(1) How do an individual's beliefs about the rational choice factors in regarding to the personalization advertising services affect consumers' reactance of such services?

(2) Are there any differences among the associations between rational choice factors and consumers' reactance of online personalization advertising based on consumers' different goals to surf the IT platforms (e.g. searching and browsing)? 
(3) How do feeling factors affect an individual's beliefs about the rational choice factors in regarding to the personalization advertising?

Drawing on the theory of rational choice [11], we identified the rational choice factors in the context of online personalized advertising and then tested how they affect consumers' reactance. Secondly, we proposed two critical feeling factors, curiosity and vulnerability, and examined the relations between rational choice factors and them.

The paper is organized as follows. First, we present the theoretical background of this research. This is followed by a description of the research hypothesizes, research methodology, and findings. The paper concludes with the implications of the findings and directions for future research.

\section{Theoretical Background}

\subsection{Reactance}

Reactance describes a motivation strategy that consumers use to perform against a persuasion attempt associated with a privacy invasion resulting in decreasing in the evaluation of the source of the restriction [7].

In many circumstances, the online personalized advertising can give rise to the reactance of the consumers. When perceiving inappropriately close to their preferences, the consumers' feelings of manipulated or threatened in their freedom of choice may be triggered by such personalized online service [3]. For example, when users' personality tests (e.g. esthetic choices) are predicted on the personalized advertising services, they might choose against such service. Moreover, a further antecedent of reactance is the only presentation of specific choice options which hinder consumers' evaluations of remaining alternatives [6]. Therefore, our study set out to identify the antecedents of reactance of personalized service online.

\subsection{Rational choice theory}

Rational choice theory has been widely applied to the study of individual behavior in many social and economic contexts, which demonstrates the process of an individual decision making by balancing the cost and benefit factors of his choices [11,12].

During the process of decision making, an individual recognizes alternative actional courses, and then deliberate the possible outcomes of each course. Since each person has preference for outcomes, each outcome can be associated with a perceived cost or benefit depending on how much satisfaction the outcome will produce for the individual. Therefore, overall assessment of costs and benefits of each possible course of action are shaped by individual's perceived outcomes related to the course of action [12]. As a result, an individual makes a tradeoff between the overall assessment of cost and benefit factors of courses of action to determine the best choice.
In the personalization context, we identify the personalization tradeoff from a perspective of negative affects, which refers to the balance among the rational choice factors including the perceived cost of nonpersonalization and the perceived sacrifices of the online personalized advertising.

\subsection{Searching versus browsing: The roles of consumers' goals}

Drawing on Hoffman and Novak (1996), among the main reasons for surfing the IT platforms are finding useful information and browsing for entertainment called searching and browsing respectively [13]. Based on the transactional theory, the searchers are more likely related to an efferent stance when reading the text [9]. They prefer "what will remain as the residue after the reading - the information to be acquired" ([14] p.23), rather than the enjoyment of the text, such as the rhythm and metaphors used in the reading. Moreover, searching is marked with purposive, task-specific behavior [13]. The searchers are motivated to find information to fulfill a goal of buying online.

Different from the searchers, the browsers prefer an aesthetic stance, which focuses on "what happens during the actual reading event" ([14], p.24), such as the information conveyed and its presentation. Thus, the browsers tend to approach IT platform to be entertained, rather than to quire the information [9]. Besides, browsing is a moment-bymoment activity with fewer outcomes oriented, recreational behavior [13]. The browsers are likely focus on whatever considered interesting or entertaining during site visits.

\section{Research Model and Hypotheses ${ }^{1}$}

Drawing from the literature summarized above, we designed our proposed research model from a perspective of negative effects (see Figure 1). Specially, we hypothesize that (a) the rational choice factors have salient effects on reactance of personalized online advertising service based on consumers' different goals to surf the IT platforms (e.g. searchers and browsers); (b) individual feeling factors jointly influence the rational choice factors.

\subsection{The role of rational choice factors on consumers' reactance of online personalized advertising for consumers' goals}

Amazon.com, Facebook, and other personalized online advertising service platforms provide users with related information or products, specific consuming incentives, consumers' personal interest and enjoy interactions. However, if not choosing online personalized advertising, the consumers might be trapped in information overload

\footnotetext{
${ }^{1}$ Because of the insignificance of the relationship between vulnerability and perceived costs of non-personalization and no sufficient theoretical support for such relationship, we would not intend to study the hypothesis regarding the relationship between vulnerability and perceived costs of nonpersonalization.
} 
with much irrelevant information and more time cost on accessing the promotable information and making decisions
[5]. Recent studies have also confirmed that online personalized advertising could facilitate consumers

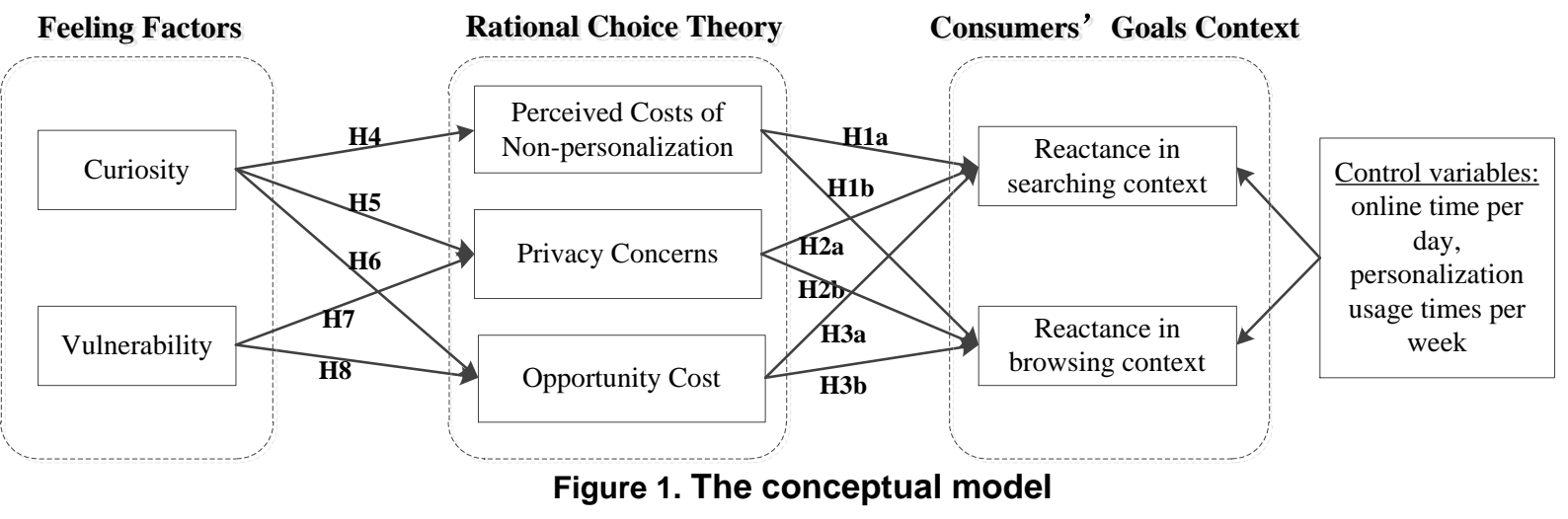

online shopping [3]. Therefore, we proposed a concept of the cost of non-personalization to reflect the cost or sacrifice of consumers' online shopping without the support of online personalized advertising. As such, in the personalization context, the more cost the consumers perceived if not using the personalized services, the more likely they prefer the personalized services with less reactant. Hence, we posit:

H1a: The perceived costs of the non-personalization will have a negative effect on consumers' reactance of online personalized advertising when consumers' goals are to search.

H1b: The perceived costs of the non-personalization will have a negative effect on consumers' reactance of online personalized advertising when consumers' goals are to browse.

Most of IS literatures have defined the concept of privacy concern as individuals' worries about potential loss of control over personal information [15]. Since online personalization platforms rely on large amounts of personal data about the consumers in order to offer users more targeted and convenient services, individuals' control over personal information would be lost in an accidental or deliberate way. Misuse of individual local information and other personal information may discover and track users' identity and behavior in unknown parties, price discrimination or unauthorized access. As such, In the personalization context, the consumers may be reactant with the online personalized advertising, when experiencing privacy concerns about the privacy collection on personalized banners [16]. Hence, we posit:

H2a: Privacy concerns will have a positive effect on consumers' reactance of personalized online advertising service when consumers' goals are to search.

H2b: Privacy concerns will have a positive effect on consumers' reactance of personalized online advertising service, when consumers' goals are to browse.

Research has indicated that tailored offerings were presented to the consumers only the things that they liked or the offerings assumed they liked, which could give rise to much opportunity cost of foregoing alternative service offerings [6]. This issue contributes to the overall opportunity costs of foregoing alternative service offerings, which is defined as the sacrifices of the highest benefits of alternative service context. The personalized advertising services have limited individual possibilities to choose, and might inhibit their capacity to make informed decisions on what they buy and even what they think [6]. The consumers might feel manipulated or threatened in their freedom of choice. In the personalization context, the more opportunity cost of the online personalized advertising the consumer realize, the more they may be reactant with those services. Hence, we posit:

H3a: Opportunity cost will have a positive effect on consumers' reactance of online personalized advertising when consumers' goals are to search.

H3b: Opportunity cost will have a positive effect on consumers' reactance of online personalized advertising when consumers' goals are to browse.

\subsection{The role of individual feelings in rational choice factors}

In this study, we focus on the influences of curiosity and vulnerability feelings on the perceived beliefs of personalization advertising services.

The former involves in increased positive feeling which is influenced by the visual, auditory, or tactual perceptions of the novel personalized characteristics at the early stage of the interaction with the personalized advertising services [17, 18]. Clore and Gasper (2000) indicated that individuals in positive feeling states would tend to seek more positive evidence that confirms their feelings than people in a negative feeling [19]. In the personalization context, the feeling of curiosity is associated with positive evaluation of personalization value of consumers' personal interest and enjoyable interactions, triggering more perceived cost of non-personalization. Hence, we posit:

H4: Curiosity feeling will have a positive effect on the perceived costs of the non-personalization.

The feeling of curiosity pertains to the personality of openness to try new things and experience new situations [20]. Junglas et al. (2008) suggested that individual high on openness intend to develop a broader and deeper sense of awareness, which are more sensitive to things that are 
threatening than those who are low on openness [20]. When consumers become more curious through their transaction with a highly personalized advertising, they might sense greater threaten. As such, in the personalization context, the more curiosity the individuals perceive, the more they are likely to consider things as harmful. Hence, we posit:

H5: Curiosity feeling will have a positive effect on privacy concerns.

As pointed by Kashdan and Roberts (2004), curiosity is uniquely related to the development of interpersonal relationship with strangers [21]. The consumers with high curiosity might be more responsive and infuse more novel twists of excitement into interactions, which is associated with the behavior of seeking and capitalizing on the interaction with their partner [21]. As such, the personalized advertising services' stimulating features which would cause individual curiosity can ensure an appropriate level of a long-term communication with the online users. In the personalization context, greater curiosity is starting point of more focus on the interactions with the online personalized advertising and less access to alternative service offerings, which results in more perceived opportunity cost of the online personalized advertising. Hence,

H6: Curiosity feeling will have a positive effect on the opportunity cost.

Vulnerability is related to a state of mind in which an individual feels lack of control over the situation and experiences a state of powerlessness [22]. Such vulnerability feeling arises when the tailored information exchange with online personalized advertising prompts the consumers to feel exploited lacking of control over their personal information [22]. Such an experience is related to strong feeling intensity, caused by the intense threat to their selfconcept, so that the individual could concern as if her or his security and well-being were at risk [22]. The relationship between vulnerability and privacy concerns is welldocumented for general online personalization, which suggests that the feeling of vulnerability may shape privacy concerns [3]. Hence, we posit:

H7: Vulnerability feeling will have a positive effect on privacy concerns.

Recent research also indicates that vulnerability is so undesirable that consumers avoid objects associated with this vulnerability feeling [3]. In the personalization context, we thus posit that when consumers perceive greater vulnerability in the latter interactions, they prefer to avoid accessing this online personalized advertising. As such, the collections of some alternative advertising services might also produce strong negative feelings because of the effects of similarity on one's appraisal [23]. Therefore, the consumers might forego the highest benefits of alternative service offerings, which may increase the opportunity costs [6]. Hence, we posit:

H8: Vulnerability feeling will have a positive effect on the opportunity cost.

\section{Research Method and Data}

\subsection{Instrument development}

Measures in our study are primarily adapted from previously validated scales, and multi-item scales are used to improve the reliability and validity of the measurement. They are then rewarded to fit the context of our study. The measurement scales for curiosity feeling are adapted (four items) from Litman and Spielberger (2003), whereas the items for vulnerability are adapted (three items) from Aguirre et al. (2015) [3,14]. For the perceived cost of nonpersonalization (three items), we adapt the measures of noncompliance from Bulgurcu et al. (2010) and revise their original scales according to the features of nonpersonalization [5,12]. Privacy concerns (three items) are measured using the instrument suggested by $\mathrm{Xu}$ et al. (2011)[24]. Because there are no established scales available for opportunity cost, we develop the scales for this construct (three items) in line with recent research of Newell and Marabelli (2015) [6].

In order to examine the contingent effects of alternative consumers' goals on the individual perceptions and behaviors, the measures of reactance of personalized online service are designed based on Bleier and Eisenbeiss (2015) in two scenarios manipulating consumers' goals (e.g. searching and browsing), respectively [16]. Based on Schlosser et al. (2006), the searchers are instructed to "go shopping purposefully", while they are instructed to "have fun, looking at whatever you consider interesting and/or entertaining" in the browsing scenario [9]. Each of the participants is required to answer the questions under both of the two scenarios.

All constructs are measured using multi-items with sevenpoint Likert scales by asking the participants to respond to the designed items of the constructs according to the extent to which they agree with them. The Likert scale ranges from 1 to 7, on which 1 represents "strongly disagree" and 7 represents "strongly agree". Seven-Likert scale is chosen in our study since it is the most widely used psychometric scale in survey research.

In order to fully account for the differences among the participants, two control variables that may affect reactance are added to this study, including online time per day and personalization usage times per week. The constructs and measurement items are seen in Table 1and Table 2.

\subsection{Data collection}

This proposed model is test empirically with data collected through an individual level survey instrument. The samples were randomly collected through social medias, such as QQ and WeChat. Each participant was asked to assume both searching and browsing contexts, and then complete the questionnaire. Since the target participants were in China, we carried out questionnaire translation and back-translation between English and Chinese carefully by two translators.

Before the final field study, we conducted a pilot study with 60 participants. This pilot study was to ensure that the 
procedures of field study were well communicated and understood, as well as identifying and refining potentially ambiguous measurement items in the research model. The reliability of measurement items for each construct are assessed using Cronbach's $\alpha$, and convergent and discriminant validity are assessed using principal components analysis. Both assessments yielded acceptable results in almost all instances. Measurement items with unacceptably low Cronbach's $\alpha$ were rephrased or dropped [25].

\section{Table 1. The definition of the constructs}

\begin{tabular}{|l|l|}
\hline \multicolumn{1}{|c|}{ Construst } & \multicolumn{1}{c|}{ Definition } \\
\hline Curiosity Feeling (CF) & $\begin{array}{l}\text { Refers to a positive emotional motivation system aroused by novel, complex, or ambiguous stimuli } \\
\text { from the dominant features of online personalized advertising }\end{array}$ \\
\hline Vulnerability Feeling (VF) & $\begin{array}{l}\text { Refers to a state of mind in which an individual feels lack of control over the situation and experiences } \\
\text { a state of powerlessness when using online personalized advertising }\end{array}$ \\
\hline $\begin{array}{l}\text { Perceived costs of the non- } \\
\text { personalization (NPC) }\end{array}$ & $\begin{array}{l}\text { Refers to the perceived cost or sacrifice of consumers' online shopping without the support of online } \\
\text { personalized advertising }\end{array}$ \\
\hline Privacy concerns (PCO) & $\begin{array}{l}\text { Refers to individuals' worries about potential loss of control over personal information when using } \\
\text { online personalized advertising }\end{array}$ \\
\hline Opportunity cost (OC) & Refers to the sacrifices of the highest benefits of alternative service context \\
\hline $\begin{array}{l}\text { Reactance in searching context } \\
\text { (REA-S) }\end{array}$ & $\begin{array}{l}\text { Refers to a motivation strategy that consumers use to perform against a persuasion attempt to online } \\
\text { personalized advertising when their goals are to search }\end{array}$ \\
\hline $\begin{array}{l}\text { Reactance in searching context } \\
\text { (REA-B) }\end{array}$ & $\begin{array}{l}\text { Refers to a motivation strategy that consumers use to perform against a persuasion attempt to online } \\
\text { personalized advertising when their goals are to browse }\end{array}$ \\
\hline
\end{tabular}

Table 2. Constructs and measurement items

\begin{tabular}{|c|c|}
\hline Construct & Measurement Items \\
\hline $\begin{array}{l}\text { Curiosity Feeling } \\
(\mathrm{CF})\end{array}$ & I am interested in the online personalized advertising on A.com. \\
\hline \multirow{3}{*}{$\begin{array}{l}\text { Vulnerability } \\
\text { Feeling (VF) }\end{array}$} & The online personalized advertising on A.com makes me feel exposed. \\
\hline & The online personalized advertising on A.com makes me feel unprotected. \\
\hline & The online personalized advertising on A.com makes me feel unsafe. \\
\hline $\begin{array}{l}\text { Perceived costs of } \\
\text { the non- } \\
\text { personalization } \\
\text { (NPC) }\end{array}$ & I believe that using the non-personalization besides A.com would create disadvantages for me. \\
\hline \multirow{3}{*}{$\begin{array}{l}\text { Privacy concerns } \\
\text { (PCO) }\end{array}$} & I am concerned that the information I submit to A.com could be misused. \\
\hline & I am concerned that others can find private information about me from A.com. \\
\hline & I am concerned about providing personal information to A.com, because it could be used in a way I did not foresee. \\
\hline $\begin{array}{l}\text { Opportunity cost } \\
\text { (OC) }\end{array}$ & $\begin{array}{l}\text { When using the online personalized advertising on A.com, I am concerned that the IT platform offering online } \\
\text { personalized advertising is determining what I see. }\end{array}$ \\
\hline \multirow{5}{*}{$\begin{array}{lr}\text { Reactance } & \text { in } \\
\text { searching } & \text { context } \\
\text { (REA-S) } & \end{array}$} & When I go shopping purposefully, I believe that: \\
\hline & The online personalized advertising on A.com is forced upon me. \\
\hline & The online personalized advertising on A.com is unwelcomed. \\
\hline & The online personalized advertising on A.com is interfering. \\
\hline & The online personalized advertising on A.com is intrusive. \\
\hline \multirow{5}{*}{$\begin{array}{lr}\text { Reactance } & \text { in } \\
\text { browsing } & \text { context } \\
\text { (REA-B) } & \end{array}$} & When I have fun, looking at whatever I consider interesting and/or entertaining, I believe that \\
\hline & The online personalized advertising on A.com is forced upon me. \\
\hline & The online personalized advertising on A.com is unwelcomed. \\
\hline & The online personalized advertising on A.com is interfering. \\
\hline & The online personalized advertising on A.com is intrusive. \\
\hline
\end{tabular}

There are a total of 301 individuals were recruited for our study. The recruiting message confirmed that the participants were being recruited for a study examining online personalized advertising. Because the participation was voluntary, some respondents submitted only partially filled questionnaires that we subsequently eliminated. Finally, a total of 281 valid responses were received. Specific demographic information of participants is given in Table 3 .

Table 3. Respondent demographics

\begin{tabular}{|l|l|l|}
\hline $\begin{array}{c}\text { Demographic } \\
\text { variables }\end{array}$ & \multicolumn{1}{|c|}{ Category } & Count (percent) \\
\hline \multirow{3}{*}{ Gender } & Female & $109(38.8 \%)$ \\
\cline { 2 - 3 } & Male & $172(61.2 \%)$ \\
\hline \multirow{3}{*}{ Age } & $15-25$ & $128(45.6 \%)$ \\
\cline { 2 - 3 } & $25-35$ & $144(51.2 \%)$ \\
\cline { 2 - 3 } & 35 and over & $9(3.2 \%)$ \\
\hline Online time per & 1 hour and below & $16(5.7 \%)$ \\
\hline
\end{tabular}




\begin{tabular}{|l|l|l|}
\hline \multirow{4}{*}{ day } & $1 \sim 5$ hours & $206(73.3 \%)$ \\
\cline { 2 - 3 } & $5 \sim 10$ hours & $42(14.9 \%)$ \\
\cline { 2 - 3 } & 10 hours and over & $17(6.0 \%)$ \\
\hline \multirow{2}{*}{$\begin{array}{l}\text { Personalization } \\
\text { usage times per }\end{array}$} & Several times each day & $31(11.0 \%)$ \\
\cline { 2 - 3 } & Once to twice per day & $58(20.6 \%)$ \\
\cline { 2 - 3 } & Once to twice each week & $79(28.1 \%)$ \\
\cline { 2 - 3 } & Once to twice each month & $61(21.7 \%)$ \\
\cline { 2 - 2 } & Other & $52(18.5 \%)$ \\
\hline
\end{tabular}

\section{Data Analysis and Results}

Our study involves investigating the personalization paradox from a perspective of negative effects. Recent research has confirmed that PLS is suitable for accommodating the relative complex relationships among various constructs by avoiding inadmissible solutions and factor indeterminacy [24]. Thus, the relationship the constructs in our research model are tested using PLS.

\subsection{Measurement model}

The measurement model is evaluated by examining the convergent validity and discriminant validity of the research instrument. The three-step approach was followed to determine convergent validity of measured reflective constructs in a single instrument: Cronbach's alpha, composite reliability of constructs, and average variance extracted by constructs. The results are presented in Tables
4 and 5 . The item reliability was assessed by examining the loading of each item on the construct, and found that the reliability score for all the items exceeded 0.8 (See in Table 5). As shown in Table 4, the composite reliabilities constructs with multiple indicators are greater than or equal to 0.9 and Cronbach's alpha value are greater than or equal to 0.8 , which exceeded the criterion of 0.7 [26]. The average variances extracted for the constructs are all above the criterion of $50 \%$. These results demonstrate that all constructs have adequate reliability scores, supporting the convergent validity of the measurement model.

To ensure the discriminant validity of constructs, the square root of the variance between a construct and its measures should be greater than the correlations between the construct and any other construct in the research model. Table 4 reports the diagonal to the non-diagonal elements. Besides, item loadings on their own construct are significantly higher than the cross-loadings on any other construct (see Table 5) [27]. These results show that all items in our construct met the requirement of discriminant validity.

Finally, because all data are self-reported in our empirical study, a test for common method bias is conducted based on the method proposed by Chin et al. (2012) and Armstrong et al. (2015) [28, 29]. Overall, most method factor loadings are not significant. Based on our test, the results suggest that the common method bias is not a serious concern in our study.

Table 4. Latent variable correlations and discriminate validity

\begin{tabular}{|c|c|c|c|c|c|c|c|c|c|c|}
\hline & $\begin{array}{c}\text { Composite } \\
\text { reliability }\end{array}$ & $\begin{array}{c}\text { Cronbach's } \\
\text { alpha }\end{array}$ & $\begin{array}{c}\text { Variance } \\
\text { Extracted }\end{array}$ & CF & VF & NPC & PCO & OC & REA-S & REA-B \\
\hline VF & 0.942 & 0.908 & 0.845 & -0.009 & 0.919 & & & & & \\
\hline $\mathrm{PCO}$ & 0.951 & 0.922 & 0.865 & 0.106 & 0.519 & -0.096 & 0.930 & & & \\
\hline $\mathrm{OC}$ & 0.907 & 0.846 & 0.765 & 0.245 & 0.383 & 0.085 & 0.605 & 0.874 & & \\
\hline REA-S & 0.903 & 0.858 & 0.701 & -0.071 & 0.604 & -0.152 & 0.507 & 0.493 & 0.837 & \\
\hline
\end{tabular}

Table 5. Item loadings and cross-loadings

\begin{tabular}{|c|c|c|c|c|c|c|c|}
\hline Indicator & CF & VF & NPC & PCO & OC & REA-S & REA-B \\
\hline CF1 & 0.854 & 0.077 & 0.347 & 0.180 & 0.310 & 0.057 & 0.111 \\
\hline CF2 & 0.885 & -0.051 & 0.445 & 0.096 & 0.188 & -0.106 & -0.044 \\
\hline CF3 & 0.892 & -0.085 & 0.529 & -0.004 & 0.132 & -0.140 & -0.005 \\
\hline CF4 & 0.806 & 0.042 & 0.370 & 0.103 & 0.223 & -0.047 & 0.015 \\
\hline VF1 & 0.087 & 0.890 & 0.0170 & 0.422 & 0.325 & 0.519 & 0.471 \\
\hline VF2 & -0.018 & 0.936 & -0.082 & 0.512 & 0.376 & 0.589 & 0.496 \\
\hline VF3 & -0.082 & 0.930 & -0.096 & 0.489 & 0.351 & 0.553 & 0.505 \\
\hline NPC1 & 0.483 & -0.005 & 0.925 & -0.082 & 0.110 & -0.108 & 0.015 \\
\hline NPC2 & 0.456 & -0.056 & 0.946 & -0.059 & 0.090 & -0.145 & -0.032 \\
\hline NPC3 & 0.449 & -0.115 & 0.935 & -0.128 & 0.036 & -0.173 & -0.104 \\
\hline PCO1 & 0.105 & 0.519 & -0.073 & 0.938 & 0.551 & 0.496 & 0.397 \\
\hline PCO2 & 0.101 & 0.459 & -0.125 & 0.930 & 0.584 & 0.458 & 0.333 \\
\hline PCO3 & 0.090 & 0.467 & -0.071 & 0.923 & 0.557 & 0.459 & 0.369 \\
\hline OC1 & 0.220 & 0.401 & 0.082 & 0.554 & 0.853 & 0.433 & 0.424 \\
\hline OC2 & 0.229 & 0.295 & 0.097 & 0.526 & 0.896 & 0.420 & 0.353 \\
\hline OC3 & 0.192 & 0.299 & 0.042 & 0.503 & 0.873 & 0.439 & 0.422 \\
\hline REA-S-1 & -0.033 & 0.512 & -0.062 & 0.510 & 0.478 & 0.778 & 0.583 \\
\hline REA-S-2 & -0.097 & 0.462 & -0.195 & 0.372 & 0.429 & 0.856 & 0.549 \\
\hline REA-S-3 & -0.049 & 0.499 & -0.148 & 0.414 & 0.371 & 0.863 & 0.635 \\
\hline REA-S-4 & -0.064 & 0.545 & -0.108 & 0.373 & 0.343 & 0.849 & 0.588 \\
\hline
\end{tabular}




\begin{tabular}{|c|c|c|c|c|c|c|c|}
\hline REA-B-1 & 0.036 & 0.493 & -0.088 & 0.435 & 0.504 & 0.633 & 0.840 \\
\hline REA-B-2 & -0.030 & 0.371 & -0.068 & 0.304 & 0.344 & 0.592 & 0.858 \\
\hline REA-B-3 & 0.036 & 0.487 & -0.016 & 0.277 & 0.303 & 0.590 & 0.850 \\
\hline REA-B-4 & 0.025 & 0.449 & -0.079 & 0.254 & 0.342 & 0.558 & 0.848 \\
\hline
\end{tabular}

\subsection{Structural model and research findings}

5.2.1. The impacts of rational choice factors on reactance: The role of consumers' goals. The hypothesis tests of the impacts of the rational choice factors on consumers' reactance of online personalized advertising are then conducted by examining the significance of the path coefficients as shown in Figure 2. We assess the explanatory power of the structural model based on the amount of variance explained in the endogenous construct (i.e. reactance). All the control variables have non-significant effects. The model without control variables in searching and browsing contexts could explain 33.3 and 24.0 percent variance of reactance, respectively, while with control variables explain 33.5 and 24.2 percent, respectively.

As seen in Figure 2, the positive impact of privacy concerns on reactance is significant in both the searching and browsing contexts $(\mathrm{H} 2 \mathrm{a}: \beta=0.290, \mathrm{P}<0.001 ; \mathrm{H} 2 \mathrm{~b}$ :

Feeling factors

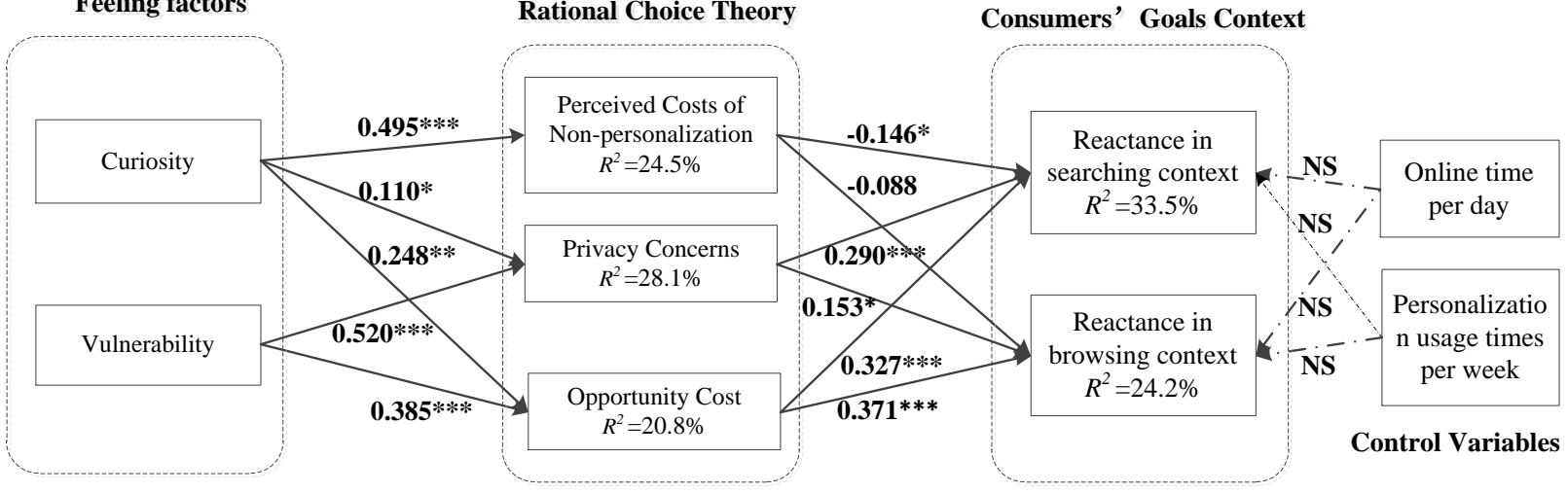

Notes: * Significant at 5\% level of significance.

** Significant at $1 \%$ level of significance.

*** Significant at $0.1 \%$ level of significance.

Figure 2. Analysis results of the relation between rational choice factors and reactance

$\beta=0.153, P<0.05$ ). Specially, the effect of privacy concern on the reactance varies upon consumers' goals, which the path coefficients decrease from 0.290 to 0.153 . The reason for such difference is due to the high information sensitivity nature of privacy concern. Studies have found that privacy concerns reflect individuals' inherent worries about their information privacy when using specific Web sites, which are strongly associated with the sensitivity of consumer-specific information the Web site is based on [30]. As such, a more text-specific individual (e.g. searcher) should be more likely to cope with higher risk inherent in his personal information, and thus should develop more negative attitudes toward the personalized services as compared to less text-specific individual (e.g. browser). However, the consumers whose goals are browsing an aesthetic stance focusing less on the text would less take the privacy concerns into account. Also, the positive relationship between opportunity cost and reactance is significant at the 0.001 level (H3a: $\beta=0.327$, $\mathrm{P}<0.001$; H3b: $\beta=0.371, \mathrm{P}<0.001$ ) in both the searching and browsing contexts. In particular, the effect of opportunity cost on the reactance for searching is little different from that for browsing, which the path coefficients increased from 0.327 to 0.371 . The reason for this issue might be ascribed to the various types of stances for browsers and searchers. Particularly, for the browsers preferring an aesthetic stance, they are predisposed to choose the experience of wide variety of new things rather than sticking with what they have previously engaged [19]. In contrast, the searchers focused on efferent stance may less rely on the diversity from the personalized services as long as the service provides the efficient information with them.

However, the proposed impact of the perceived cost of nonpersonalization on reactance is significant in the searching context at the 0.05 level (H1a: $\beta=-0.146, \mathrm{P}<0.05$ ) but insignificant in the browsing context (H1b: $\beta=-0.088)$. As to the insignificance of the effects of the perceived costs of nonpersonalization on reactance in the browsing goals, a possible explanation is because the differences among the focus of searching and browsing. Searching is marked with purposive, task-specific behavior [13]. For the searchers whose focus is shopping, it is more easily for a more tailored personalized advertising service to help them to fulfill a buying goal, compared with non-personalized advertising services. As such, the more cost the searchers perceived if not using the personalized services, the more likely they prefer the personalized services with less reactant. However, browsing is a moment-by-moment activity with fewer outcomes oriented, recreational behavior [13]. For the consumers without specific goals to buy online, they may surf the IT platforms whatever they like and might be less concerned on the availability of specific choices for buying online [9]. To the extent, the browsers would prefer to seek fulfilment of their aesthetic needs of various experiences taking no account of the inconveniences of non-personalized advertising services. 
5.2.2. The impacts of individual feeling on rational choice factors. The curiosity and vulnerability feelings can account for a substantial 24.5, 28.1 and 20.8 percent variance of the perceived cost of non-personalization, privacy concerns and opportunity cost separately. As shown in Figure 2, the feeling of curiosity is positively associated with the perceived cost of non-personalization at the 0.001 level $(\mathrm{H} 4: \beta=0.495, \mathrm{P}<0.001)$ and privacy concerns at 0.05 level $(\mathrm{H} 5: \beta=0.110, \mathrm{P}<0.05)$. In support of $\mathrm{H6}$, the positive relationship between the feeling of curiosity and opportunity cost are significant at the 0.01 level (H6: $\beta=0.248, \mathrm{P}<0.01$ ). Vulnerability is positively related to both privacy concerns and opportunity cost at the 0.001 level $(\mathrm{H} 7: \beta=0.520, \mathrm{P}<0.001 ; \mathrm{H} 8: \beta=0.385$, $\mathrm{P}<0.001)$.

\subsection{Further analyses on the relationship between reactance and click-through intention}

When the consumers experience reactance, they are motivated to restore their freedom of choice by behaving counter to the intention of a persuasion and invasion attempt [7]. As such, if gaining more reactance of the online personalized advertising, the users will refuse to click through such personalization. Also, it has been suggested in the literature that different consumers' goals holds various trusting beliefs, which might influence the consumers' behavior intentions [9]. Hence, we propose that the impact of reactance on the click-through intention in the context of different consumers' goals (e.g. searching or browsing on the IT platforms).

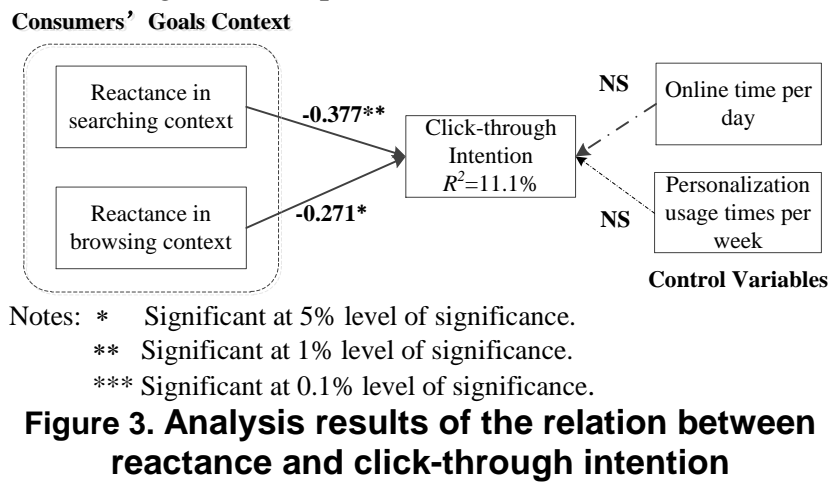

We use SmartPLS for assessing the structural model of this proposed model. The model with control variables could explain 11.1 percent variance of click-through intention. Figure 3 also indicates that the proposed impact of reactance on the click-through intention is significant in the searching goals at the 0.05 level $(\beta=-0.377, \mathrm{P}<0.01)$ and in the browsing goals $(\beta=-0.271, \mathrm{P}<0.5)$.

A plausible explanation for the different significance of reactance in searching and browsing context on clickthrough intention is that the diverse trust beliefs inherent in the searchers and browsers. As an example, for the consumers preferring to search on the IT platform, they focus on integrity beliefs, which reflect the trust perceptions about the firms' morel standards, regardless of how it feels about the individuals [9]. Therefore, based on the expectancy-value theory of attitude, the searchers would choose to not click through the personalized service in response to reactance with the feelings of manipulated or threatened in their freedom of choice.

In contrast, the browsers' are likely more personal and will be accompanied by benevolence beliefs which are the most personal aspects of trust [9]. Benevolence beliefs reflect consumers' beliefs that the firm cares about their welfare and well-being. As such, the browsers may trust that the personalization firms are concerned about their own preferences, even if doing so results in their profit reductions. Thus, although the personalized advertising services make them feel controlled of their freedom of choice, the browsers may not worry too much about reactance and intent to click on the personalized advertising services.

\section{Discussion}

\subsection{Theoretical implications}

This study strives to further extend prior research on the personalization paradox in several ways. First, this study attempts to understand personalization paradox from a perspective of negative effects by looking into the consumers' reactance. Most of the previous studies on the personalization paradox intent to research on the consumers' willingness to disclosure or adoption of the personalization from a positive perspective $[19,23]$. However, empirical studies indicated that a large majority of consumers still do not want the retailers to adjust targeted advertisements to their online behaviors across websites. Although Aguirre at al. (2015) suggest various kinds of personalized advertising services might trigger reactance, it pays little attention on the influencing mechanism on consumers' reactance of online personalized advertising [3]. In this paper, we focus on studying consumers' reactance of online personalized advertising from the perspective of negative effects.

Second, this study empirically unpacks and validates an updated conceptualization of personalization paradox (e.g. perceived cost of non-personalization, privacy concern and opportunity cost). The concepts of personalization paradox in existing literatures mainly only focus on the tension between consumers' great values in receiving customized applications and their growing concerns about information privacy $[3,19]$. Although Xu et al. (2011) suggest that a more comprehensive examination of risks that affect value perceptions is needed, the examination of extant concept is restricted only to privacy [24]. Newell and Marabelli (2015) indicated that the personalization services have strong negative effects on the consumers' possibility to choose and their capacity to make informed decisions [6]. Thus, this research goes beyond unveiling the personalization paradox by examining the balance of benefits and risks of personalization advertising services extending to opportunity cost through the perspective of rational choice theory.

Third, this study provides theoretical supports for the influence of feeling effects on the rational choices factors in 
the personalization paradox context. Even though the information systems adoption literature and the underlying theories suggests the service influences on the consumers is dynamically associated with the impression amounts of the IT platforms [16], less attention has been poured to situational factors of IT platforms reflected by individual feeling factors. To assess the dynamic influences of the situational features of the IT platforms, our study seeks to identify the two individual feeling factors. Fristly, The feeling of individual curiosity triggered by visual or auditory perceptions of personalized online service platforms may be the primary driver of the influence of perceived beliefs. Secondly, vulnerability feeling acts as another crucial set of drivers of perceived beliefs. Our results indicate that curiosity and vulnerability have significant influences on the rational choice factors.

Finally, our findings demonstrate that the influences of the rational choice factors on reactance vary based on different consumers' goals (e.g. searching or browsing). Recent research has shown that the roles of the consumers' goals for surfing IT platforms have yielded distinct psychological appeals of the Web sites to consumers [9]. However, in the context of personalization paradox, there are no studies focusing on examining such issue. In this study, we provide new insight into examining the roles of consumers' goals in the relationship between the rational choice factors and reactance. It suggests that the impacts of the rational choice factors on consumers' reactance of online personalized advertising are all significant in the searching context, while only the impact of opportunity cost on consumers' reactance of online personalized advertising is significant in the browsing context.

\subsection{Practical implications}

In addition to the theoretical contributions, our research also suggests how online retailers who provide personalized advertising for the users could effectively improve their performance. Firstly, the results provide the online retailers with some recommendations in terms of consumers' positive and negative feelings based on IT platforms' content, which in turn influence their perceptions of the personalization paradox. As an example, the online retailers should make IT platform more attractive in order to increase the individual curiosity, while individual guarantee measure should be also taken on the IT platforms, which might decrease the consumers' feeling of vulnerability.

Secondly, it is essential for the online retailers pay more attention to the rational choice factors in response to the personalization paradox. For example, in order to reduce consumers' reactance of online personalized advertising, the retailers should improve their tailored abilities to fulfil various service demands of the consumers, which may make the consumers rely on them. Also, the retailers should consider effective information privacy assurances to decrease the consumers' privacy concerns. Moreover, for declining the individual perceived opportunity cost, more alternative personalized advertising services should be also provided with the consumers to choose.

Finally, the online retailers providing personalized advertising should be aware of two different types of consumers' goals on surfing IT platforms since these goals may influence consumers' perceptions on the IT platforms in different ways. Our results indicated that the measures for the perceived costs of non-personalization and privacy concerns are effective in reducing consumers' reactance of online personalized advertising in the searching context. Thus, if most visitors are searcher, the retailers should enhance the advertising's tailored abilities to increase the perceived cost of non-personalization and take actions to reduce the consumers' privacy concerns, but if most visitors are browsers, such investments may be ineffective. Retailers can identify the segment visiting their sites by using clickstream data, usability studies, or some traditional market research methods.

\subsection{Limitations and future studies}

Despite several theoretical and practical contributions in our study, findings are considered in light of limitations, which offer potential future research directions. Frist, since this study only examined a subset of individual feeling factors at a specific level, future studies would investigate the other feature factors of IT platforms. For instance, effort could be devoted to examining the effects of legislative privacy policy and guarantee offered by IT-enabled personalization service providers. It is feasible that legislative privacy policy is more effective than guarantee in enhancing privacy protection belief. Besides, the consumers' goals in the study represent a simplification of all the searching and browsing goals, which may limit the generalizability of our findings. Future work could examine the applicability of our findings to other different consumers' reasons for surfing the IT platforms. Moreover, the potential moderating effect of consumers' goals among the relationship between the ration choice factors and reactance would be tested in the future work, as well as some other possible moderator between these factors (e.g. social influence).

\section{Conclusion}

Since reactance is an important management issue in the personalization context, this study attempts to extend our knowledge about the personalization paradox from the perspective of negative effects by studying consumers' reactance of online personalized advertising. To be specific, we identified the rational choice factors rooted in the rational choice theory in the context of reactance and tested their impacts on reactance, with consideration of individual feeling factors in a specific situation. Moreover, our results provided some preliminary evidences to indicate that different consumers' goals (e.g. searching and browsing) may have contingent effects on the associations between rational choice factors and reactance. Using survey data and structural equation modelling, we test the hypotheses on how individual feelings could influence rational choice factors, and how these 
rational choice factors affect consumers' reactance of online personalized advertising. The hypothesized relationships are generally supported by the data. We confirm that curiosity and vulnerability could significantly influence the rational choice factors, and the effects of the rational choice factors on consumers' reactance of online personalized advertising are significant in the searching goal while partly significant in the browsing goal. We believe the results of this study will provide instrumental insights for online retailers who provide the users with personalized advertising to improve their performance effectively.

\section{Acknowledgement}

This research was funded by the grants from the National Natural Science Foundation of China (\#71472053, \#71429001, \#71201039), and a grant from the Ph.D. Programs Foundation of Ministry of Education of China (\#20132302110017), and the grants from the Postdoctoral Science Foundation of China (\#2014M550198, 2015T80363).

\section{References}

[1] G. Hawkings, "Will Big Data Kill All But the Biggest Retailers?”, Journal, Harvard Business Review,2012.

[2] A. Lambrecht, C. Tucker, "When Eoes Retargeting Work? Information Specificity in Online Advertising", Journal, Journal of Marketing Research, 50(5), 2013, pp. 561-576.

[3] E. Aguirre, D. Mahr, D. Grewal, Dhruv, K. de Ruyter, M. Wetzels, "Unraveling the Personalization Paradox: The Effect of Information Collection and Trust-Building Strategies on Online Advertisement Effectiveness", Journal, Journal of Retailing, 91(1), 2015, pp. 34-49.

[4] B. Guild, "2013 Choicestream Survey: Consumer Opinions onOnline Advertising \& Audience Targeting", 2013.

[5] A. Ansari, C.F. Mela, "E-customization", Journal, Journal of Marketing Research, 40(2), 2003, pp. 131-145.

[6] S. Newell, M. Marabelli, "Strategic Opportunities (And Challenges) of Algorithmic Decision-Making: A Call for Action on the Long-Term Societal Effects of 'Datification"', Journal, Journal of Strategic Information Systems, 24(1), 2015, pp. 3-14.

[7] C.E. Tucker, "Social Networks, Personalized Advertising, and Privacy Controls", Journal, Journal of Marketing Research, 51(5), 2014, pp. 546-562.

[8] J.W. Brehm, W. Jack, "Psychological Reactance: Theory and Applications", Journal, Advances in consumer research, 16(1), 1989, pp. 72-75.

[9] A.E. Schlosser, T.B. White, B. Tiffany, S.M. Lloyd, "Converting Web Site Visitors into Buyers: How Web Site Investment Increases Consumer Trusting Beliefs and Online Purchase Intentions", Journal, Journal of Marketing, 70(2), 2006, pp. 133-148.

[10] N. F. Awad and M. S. Krishnan, "The Personalization Privacy Paradox: An Empirical Evaluation of Information Transparency and the Willingness to be Profiled Online for Personalization", Journal, MIS Quarterly, 30 (1), 2006, pp. 13-28. [11] B. McCarthy, "New Economics of Sociological Criminology", Journal, Annual Review of Sociology, 2002, pp. 417-442.
[12] B. Bulgurcu, H. Cavusoglu, I. Benbasat, "Information Security Policy Compliance: An Empirical Study of Rationality-Based Beliefs and Information Security Awareness", Journal, MIS Quarterly, 34(3), 2010, pp. 523-A527.

[13] D.L. Hoffman, T.P. Novak, P. Thomas, "Marketing in Hypermedia Computer-Mediated Environments: Conceptual Foundations", Journal, Journal of Marketing, 1996, pp. 50-68.

[14] L. Rosenblatt, The Reader, the Text, the Poem: The Transactional Theory of the Literary Work, Southern Illinois University Press, Carbondale, 1978.

[15] N. K. Malhotra, S. S. Kim, J. Agarwal, "Internet users' information privacy concerns (IUIPC):The construct, the scale, and a causal model", Journal, Information Systems Research, 15(4), 2004, pp. 336-355.

[16] A. Bleier, M. Eisenbeiss, "The Importance of Trust for Personalized Online Advertising”, Journal, Journal of Retailing, 2015.

[17] J. A. Litman, C. D. Spielberger, "Measuring epistemic curiosity and its diversive and specific components", Journal, Journal of Personality Assessment, 80(1), 2003, pp.75-86.

[18] D.-M. Koo, S.-H. Ju, "The Interactional Effects of Atmospherics and Perceptual Curiosity on Emotions and Online Shopping Intention", Journal, Computers in Human Behavior, 26(3), 2010, pp. 377-388.

[19] G. L. Clore, K. Gasper, "Feeling Is Believing: Some Affective Influences on Belief", Emotions and beliefs: How feelings influence thoughts, 2000, pp. 10-44.

[20] I. A. Junglas, "Personality Traits and Concern for Privacy: An Empirical Study in the Context Of Location-Based Services", Journal, European Journal of Information Systems, 17(4), 2008, pp. 387-402.

[21] T. B. Kashdan, J. E. Roberts, "Trait and State Curiosity in The Genesis Of Intimacy: Differentiation from Related Constructs", Journal, Journal of Social and Clinical Psychology, 23(6), 2004, pp. $792-816$.

[22] S.M. Baker, J.W. Gentry, T.L. Rittenburg, "Building Understanding of The Domain of Consumer Vulnerability", Journal, Journal of Macromarketing, 25(2), 2005, pp. 128-139.

[23] N. H. Frijda, P. Kuipers, E. Ter Schure, "Relations among emotion, appraisal, and emotional action readiness". Journal, Journal of personality and social psychology, 57(2), 1989, pp. 212.

[24] H. Xu, X.R. Luo, J.M. Carroll, M.B. Rosson, "The Personalization Privacy Paradox: An Exploratory Study of Decision Making Process for Location-Aware Marketing", Journal, Decision Support Systems, 51(1), 2011, pp. 42-52.

[25] M. Siponen, A. Vance, Anthony, "Neutralization: New Insights into the Problem of Employee Information Systems Security Policy Violations", Journal, MIS quarterly, 34(3), 2010, pp. 487-502.

[26] J.C. Nunally, I.H. Bernstein, Psychometric Theory, New York: McGraw-Hill, 1978.

[27] W.W. Chin, W. W. Wynne "The Partial Least Squares Approach to Structural Equation Modeling", Journal, Modern methods for business research, 295(2), 1998, pp. 295-336.

[28] W. W. Chin, J. B. Thatcher, T. W. Ryan, "Assessing common method bias: problems with the ULMC technique", Journal, MIS Quarterly, 36(3), 2012, pp. 1003-1019.

[29] D. J. Armstrong, N. G. Brooks, and C. K. Riemenschneider, "Exhaustion from information system career experience: implications for turn-away intention”, Journal, MIS Quarterly, 39(3), 2015, pp. 713-727.

[30] J. Sutanto, E. Palme, C.-H. Tan, C.W. Phang, "Addressing the Personalization-Privacy Paradox: An Empirical Assessment from A Field Experiment on Smartphone Users", Journal, MIS Quarterly, 37(4), 2013, pp. 1141-1164. 